\title{
Same-different reaction times studied with a flash masking technique
}

\author{
MASAHIKO SAITO \\ Osaka City University, Osaka, Japan
}

\begin{abstract}
The reaction times (RT) needed to report whether letters were the same or different were measured using a very long exposure of the target and a very brief exposure of a flash mask. Two letters were presented simultaneously in Experiment 1. No effect of response category (same vs. different) was found, although a clear-cut luminance-dependent masking effect was present. Letter number was manipulated in Experiment 2. A three-way interaction was found among response category, number of letters, and stimulus onset asynchrony (SOA). The more difficult task produced longer RTs for "different" judgments than for "same" judgments, particularly at intermediate SOAs. These data are compared with information processing models of perception.
\end{abstract}

The same-different reaction time paradigm has been employed to investigate visual information processing. A number of explanations of such data have been proposed, including analytic models (see Egeth, 1966; Hawkins, 1969), two-process theories (e.g., Bamber, 1969; Hock, 1973; Nickerson, 1978; Silverman, 1973; Taylor, 1976; Tversky, 1969), and a "noisy operator theory" (Krueger, 1978).

In tachistoscopic recognition studies of response accuracy, many investigators have used the masking technique in order to investigate the time course of perceptual recognition. In a few studies, a reaction time (RT) measure of masking has been employed (e.g., Eriksen \& Eriksen, 1972; Eriksen \& Hoffman, 1972).

The present study examined RT measures during flash masking when the subject was asked to make same-different judgments. The masking stimulus duration was set at $50 \mathrm{msec}$. The target stimulus was presented until the subject responded. The masking stimulus was presented at a variable stimulus-onsetasynchrony (SOA) value. The subject's task was to judge whether the stimuli were the same or different. RT was measured for that judgment. This use of the brief exposure of a flash mask to study same-different judgments is different from the paradigm employed by Eriksen and Eriksen (1972) and Eriksen and Hoffman (1972): They used either a ring mask or a masking stimulus made up of letters or dots; their masking stimulus remained on until the subjects made a response; and, their subject's task was to identify a letter.

The author would like to thank Dr. Takehiro Ueno for a critical reading of his manuscript. The author is very much obliged to anonymous reviewers for their appropriate advice. Reprint requests should be sent to Masahiko Saito, Mimasaka Junior College (Mimasaka Joshi-Daigaku Tanki Daigaku-Bu), Kamigawara 32, Tsuyama-City, Okayama-Ken 708 , Japan.
The brief flash mask exposure, used in the present experiment, would be expected to lead to a temporary degradation of target contrast to minimize any contour interactions between the target and mask. Thus, the present experiment should yield data which bear on the time course of pattern discrimination.

\section{EXPERIMENT 1}

\section{Method}

Subjects. Two females and one male served as subjects. They all had normal or corrected-to-normal visual acuity in both eyes.

Stimull and Apparatus. The target stimuli used in this experiment consisted of pairs of letters simultaneously presented. The letter pairs were $N N, Z Z, N Z$, and $Z$. Each letter subtended a visual angle of $52 \times 52 \mathrm{~min}$. Letter line width was $2.8 \mathrm{~min}$. The horizontal separation between the letters was $1.0 \mathrm{deg}$. The NN and $\mathrm{ZZ}$ pairs were used to elicit a "same" response; the NZ and ZN pairs were used to elicit a "different" response.

The target stimuli and masking flashes were back-projected onto a translucent screen with Kodak Carousel projectors. The exposure durations were controlled by two electric tachistoscopic shutters. The stimuli were presented inside a rectangle that surrounded the translucent screen. The window subtended a visual angle of $5.0 \times 6.0 \mathrm{deg}$. The experimental room was illuminated at a level of $.04 \mathrm{~lx}$. The edge of the window was visible to the darkadapted eyes. The letters were presented as black lines on a white background. The luminance was $5.3 \mathrm{~cd} / \mathrm{m}^{2}$ for white background and $.16 \mathrm{~cd} / \mathrm{m}^{2}$ for the black letters. Two flash masks were used. These masks homogeneously filled the translucent screen. The luminance of the masking flash was either $22.7 \mathrm{~cd} / \mathrm{m}^{2}$ ("high") or $2.3 \mathrm{~cd} / \mathrm{m}^{2}$ ("low"). Luminances were measured with a photometer (Tektronix, J16).

Procedure. An acoustic noise warning signal was provided before the target stimulus appeared. The interval between warning signal of fset and target stimulus onset was $1 \mathrm{sec}$. The target stimulus was continuously presented until the subject responded (see Figure 1). The subjects were required to press one button whenever the two letters were identical and to press another button whenever there was no match between the letters. RT from target onset to buttonpressing response was measured. The subjects were instructed to respond as quickly and accurately as possible. The intertrial interval was about $3 \mathrm{sec}$. On the first day of the experiment, the subjects assigned one hand to the "same" button and 
the other hand to the "different" button. On the second day, hand assignment was reversed. The subjects pressed the button with their thumbs.

A flash mask was delivered to the stimulus field at a particular SOA value (see Figure 1). The SOA values were as follows: 0, 25, 50, $75,100,125,150,175$, and $200 \mathrm{msec}$. Masking flash duration was always $50 \mathrm{msec}$. In addition, no mask control condition was used.

The stimuli were presented in blocks composed of 16 trials. The four stimulus types appeared with equal frequency and were ordered randomly within each block, with the exception that no more than four target pairs in succession could be either "same" or "different."

Each block used one SOA value and one masking light intensity. Two consecutive blocks were assigned to the same SOA value, with one block having one masking light intensity and the other block the other intensity. One experimental session consisted of 19 blocks of trials ( 9 SOAs $\times 2$ masking light intensities +1 no-mask condition. Within each session, the SOA condition blocks were randomly ordered. Each subject served for four sessions, with two sessions a day. The total number of experimental trials for each subject was 1,216 . Before beginning the experimental sessions, the subjects were dark-adapted for $10 \mathrm{~min}$. The subjects also received either 128 or 64 practice trials under the no-mask condition each day.

\section{Results}

The mean RT values for correct responses ${ }^{1}$ and the error rates are shown in Figure 2. RT values that were excluded from the analysis included individual trials that gave values greater than three standard deviations above a given mean in each condition for each subject. The mean coefficient of variation of the RT values was $12 \%$.

A repeated measures analysis of variance was performed on these RTs. Since the no-mask condition did not contain two masking flash intensities, this condition was excluded from the analysis. The main effect of masking light intensity was significant $[F(1,2)=82.71, p<.05]$. High masking intensity gave longer RTs. The main effect of SOA was also significant $[F(8,16)=9.72, p<.001]$. RT declined with increasing SOA. The main effect of response category ("same" vs. "different") was not significant $(F<1)$. The main effect of subjects was significant $[F(2,108)=14.42, p<.001]$, as was the interaction between masking light intensity and SOA $[F(8,16)=10.59, p<.001]$. The intensity effect was greater at short SOAs. All other interactions were insignificant (ps $<.25$ or Fs $<1$ ).

An inspection of Figure 2 might suggest that because of the dip near an SOA of $50 \mathrm{msec}$, the RT vs. SOA functions have a cubic trend. However, this

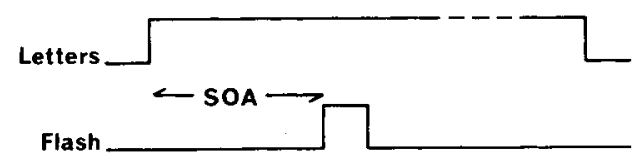

Figure 1. Timing chart of the presentation of stimuli.

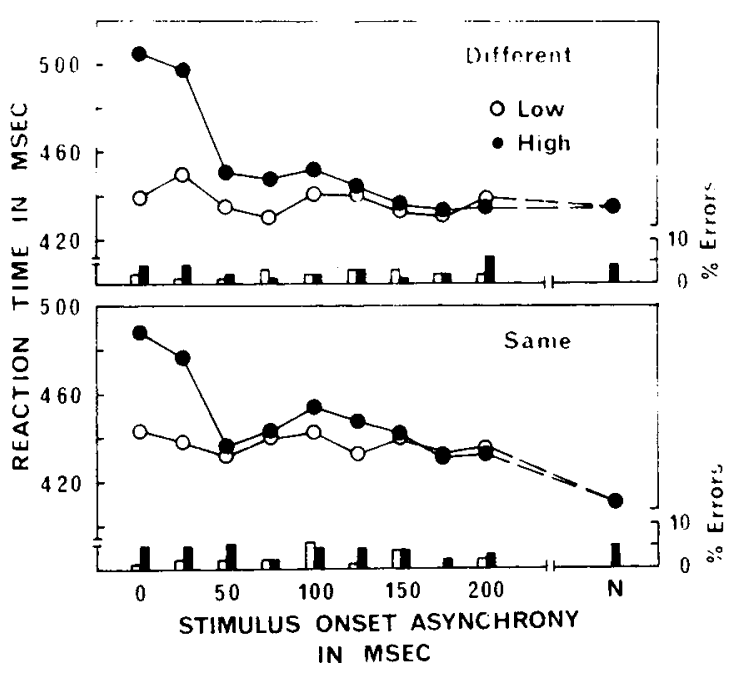

Figure 2. Mean RT values and error rates as functions of SOA and masking flash-light intensity. " $N$ " represents no-mask condition.

trend was not confirmed ${ }^{2}$ by a trend analysis $[F(1,2)$ $=1.77, \mathrm{p}>.25]$.

The error rates shown in Figure 2 are very low and show no consistent trend. The RT results therefore cannot be attributed to a speed-accuracy tradeoff.

This experiment shows that masking produces a temporary degradation of the contrast of the target stimulus that is luminance dependent. No evidence was found for the "fast same" phenomenon. However, this experiment used a simple discrimination task with only two simple letters, $\mathbf{N}$ and $\mathrm{Z}$. The failure to detect the "fast same" phenomenon might be a result of this task simplicity. In Experiment 2, therefore, the simultaneous presentation of four letters was employed in an attempt to vary task difficulty.

\section{EXPERIMENT 2}

\section{Method}

The target stimuli used in this experiment consisted of simultaneously presented two- or four-letter combinations. The letters were $\mathbf{N}$ or $Z$, as in Experiment 1. Either two or four letters were assigned to the corners of an imaginary square, which subtended a visual angle of $2.2 \mathrm{deg}$. The center of the letter was placed on the corner. In the two-letter condition, two of the four corners of the imaginary square were selected. The two letters were arranged vertically or horizontally. In this condition, the "different" stimulus had two different letters and the "same" stimulus had two identical letters. The frequencies of each such assignment were equated across experimental trials. In the four-letter condition, four letters appeared on the four corners of the imaginary square. In this condition, the "same" stimulus had four identical letters, while the "different" stimulus had three letters which were identical and one which was different from the other three. The frequencies of each such assignment were equated across experimental trials. The luminance of these stimuli was $6.0 \mathrm{~cd} / \mathrm{m}^{2}$ for the white background and $.1 \mathrm{~cd} / \mathrm{m}^{2}$ for the black letters. The experimental room was illuminated at a level of $.04 \mathrm{~lx}$. The apparatus 
was the same as that used in Experiment 1, except that two dim light dots were added to the frame of the display in vertical alignment. The separation between the dots was $6.1 \mathrm{deg}$. These dots were used to help stabilize the subject's eye fixation.

The stimuli were presented in blocks of 32 trials. Each experimental block contained every type of target stimulus. The procedure on any one trial was the same as in Experiment 1 . The target stimuli were ordered randomly within each block, except that no more than four letter assignments in succession could be either "same" or "different."

The masking condition was the same as that used in Experiment 1 , except that only the high-intensity flash (the luminance was $22.7 \mathrm{~cd} / \mathrm{m}^{2}$ ) was used.

One experimental session contained 10 blocks of trials ( 9 SOAs + 1 no-mask condition). The SOA-condition blocks were randomly ordered within each session. Each subject served for four sessions, with two sessions per day. The total number of experimental trials for each subject was 1,280 . The subjects also received 128 or 64 practice trials under the no-mask condition in each day.

The subjects were two males and female. They all had normal or corrected-to-normal visual acuity in both eyes. One male had participated in Experiment 1, but the other two subjects had not.

\section{Results}

The mean RT values ${ }^{1}$ and error rates are shown in Figure 3. The criterion for excluding abnormal RT values was the same as in Experiment 1. The mean coefficient of variation of the RT values was $13 \%$.

A repeated measures analysis of variance was done on the RTs. The results of the analysis were as follows: The main effects of both letter number and response category were insignificant $[F(1,2)=.32, p>.25$, $F(1,2)=2.74, p<.25]$, respectively. However, the main effects on both SOA and subjects were significant $[F(9,18)=8.77, p<.001$, and $F(2,120)=52.68$, $\mathrm{p}<.001$, respectively]. The interactions between response category and subjects and between SOA and subjects were both significant $[F(2,120)=17.58$, $\mathrm{p}<.001$, and $\mathrm{F}(18,120)=3.44, \mathrm{p}<.001$, respectively]. The interaction between letter number and re-

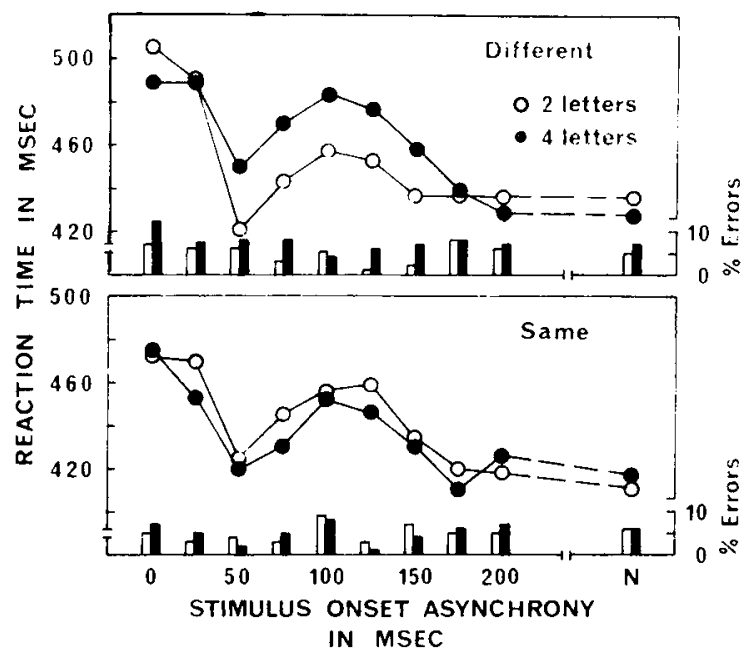

Figure 3. Mean RT values and error rates as functions of SOA and number of letters. " $N$ " represents no-mask condition. sponse category was insignificant $[F(1,2)=14.59$, $\mathrm{p}<.10$ ], as were the other two-way interactions (ps $>.25$ ). But the three-way interaction among letter number, response category, and SOA was significant $[F(9,18)=2.47, p<.05]$. The other three-way interactions and a four-way interaction were all insignificant (Fs $<1$ ).

These results confirm the results of Experiment 1. They also show a weak "fast same" effect, as evidenced by the significant three-way interaction. This means that "same" judgments are significantly faster than "different" judgments, but mainly at intermediate SOAs. For example, at an SOA of $100 \mathrm{msec}$, judgments of "same" give an RT of $452 \mathrm{msec}$ and "different" judgments give an RT of $483 \mathrm{msec}$ when four letters are used. When two letters are used, "same" judgments give an RT of $456 \mathrm{msec}$ and "different" judgments give an RT of $457 \mathrm{msec}$. A smaller effect is found near zero SOA, and the effect is gone at long SOAs.

Although the RT vs. SOA functions given in Figure 3 look like they might follow a cubic curve, a trend analysis did not confirm the significance ${ }^{2}$ of that effect $[F(1,2)=2.50, p>.25]$.

\section{DISCUSSION}

In Experiment 1, no "fast same" effect was found. On the other hand, the three-way interaction among letter number, response category, and SOA found in Experiment 2 shows a weak "fast same" effect, when task difficulty is increased. These findings may be interpreted within the framework of Krueger's (1978) "noisy operator theory."

Krueger (1978) argued that the information given to the information processor is noisy and that such internal noise is more likely to produce spurious featural mismatches than matches. Also, more recheckings would be needed to remove these spurious mismatches for "different" responses than for "same" responses. (The basis of the prediction is that the probability distribution of the noise is skewed; see Krueger, 1978). Now a given mean RT value would be an expression of the number of recheckings, and hence the mean "different" RT would be slower than mean "same" RT: this is the "fast same" phenomenon. If this were the case, we could interpret the results of Experiment 1 as follows: (1) If the difference between the letters $\mathbf{N}$ and $Z$, employed in the present study, was rather great, then only a few recheckings would be needed. In this situation, we would not find the "fast same" phenomenon, because the phenomenon depends upon the number of recheckings. (2) When the masking flash appears at an SOA, the effect of the noise would temporarily increase, since the figure-ground contrast would decrease. But, if the difference between two letters is large enough, the 
number of recheckings would not appreciably increase, even in the presence of the masking flash for a short period.

On the other hand, in Experiment 2, four letters were presented simultaneously; three identical letters and only one different letter were contained in the four-letter combinations assigned to the "different" stimulus. This suggests that the perceptual difference between "same" and "different" combinations is smaller for the four-letter condition than for the twoletter condition. This implies that the discrimination is therefore more difficult. In such a case, Krueger's (1978) model would predict that slower "different" responses should occur when the masking flash decreases the figure-ground contrast. At very long SOAs, processing would have already ended and there would be no reason to expect a difference. Thus, a three-way interaction among response category, letter number, and SOA would be found.

Many experimental results (e.g., Bamber, 1969; Hock, 1973; Nickerson, 1978; Silverman, 1973; Taylor, 1976; Tversky, 1969) have suggested that analytic models (see Egeth, 1966; Hawkins, 1969) have difficulty with the "fast same" phenomenon (see Nickerson, 1978). A number of two-process theories have been proposed to explain this phenomenon. However, a two-process theory which assumes the existence of an "identity reporter" and a "serial processor" (e.g., Bamber, 1969; Hock, 1973; Nickerson, 1978; Silverman, 1973; Taylor, 1976; Tversky, 1969) would have more difficulty with the results of Experiment 2, because such a theory does not have properties which predict the behavior of "identity reporter" and "serial processor" when figure-ground contrast or noise level is altered. However, such theories might be modified by incorporating such properties.

Finally, we must comment on the masking function shape. Although the trend analyses failed to confirm this, ${ }^{2}$ the masking functions in Experiments 1 and 2 seem to dip near an SOA of $50 \mathrm{msec}$. Observing the stimulus field, we could detect the flash onset at long SOAs. But, at short SOAs, we could not appreciate the onset that would be integrated completely with the target stimulus. The difference between the impressions of visual field at each SOA might affect the function shape to some degree by altering the processing speed, vigilance, or response set. In addition, it was found that the summation index of two light pulses varies in a nonmonotonic manner against SOA values (Ueno, 1977; Uetsuki \& Ikeda, 1970), and that the intratarget metacontrast effects shows the nonmonotonic function shape (Brussel \& Favreau, 1977). The experimental intensities and durations of stimulus exposure and adaptation levels for illumination employed by those investigators were very different from those of the present study. However, the nonsignificant dip observed in
Experiments 1 and 2 might be a small residual of the dips that these investigators found under more favorable circumstances.

\section{REFERENCES}

Bamber, D. Reaction times and error rates for "same".-"different" judgments of multidimensional stimuli. Perception \& Psychophysics, 1969, 6, 169-174.

Brussel, E. M., \& Favreau, O. E. Backward pattern masking can vary as a nonmonotonic function of target duration: On the influence of intratarget metacontrast. Journal of Experimental Psychology: Human Perception and Performance, 1977, $3,461-472$.

Egeth, H. E. Parallel and serial processes in multidimensional stimulus discrimination. Perception \& Psychophysics, 1966, 1, 245-252.

Eriksen, C. W., \& Eriksen, B. A. Visual backward masking as measured by voice reaction time. Perception \& Psychophysics, $1972,12,5-8$.

Eriksen, C. W., \& Hoffman, J. E. Temporal and spatial characteristics of selective encoding from visual displays. Perception \& Psychophysics, 1972, 12, 201-204.

Hawkins, H. L. Parallel processing in complex visual discrimination. Perception \& Psychophysics, 1969, 5, 56-64.

Носк, H. S. The effect of stimulus structure and familiarity on same-different comparison. Perception \& Psychophysics, 1973, 14, 413-420.

KrUeger, L. E. A theory of perceptual matching. Psychological Review, 1978, 85, 278-304.

Nickerson, R. S. On the time it takes to tell things apart. In J. Requin (Ed.), Attention and performance VII. Hillsdale, N.J: Erlbaum, 1978.

Silverman, W. P. The perception of identity in simultaneously presented complex visual displays. Memory \& Cognition, 1973, $1,459-466$.

TAYLOR, D. A. Holistic and analytic processes in the comparison of letters. Perception \& Psychophysics, 1976, 20, 187-190.

TVE RsKy, B. Pictorial and verbal encoding in a short-term memory task. Perception \& Psychophysics, 1969, 6, 225-233.

UENo, T. Temporal characteristics of the human visual system as revealed by reaction time to double pulses of light. Vision Research, 1977, 17, 591-596.

Uetsuki, T., \& ÍEDA, M. Study of temporal visual response by the summation index. Journal of the Optical Society of America, 1970, 60, 377-381.

\section{NOTES}

1. When the subjects pressed the buttons twice at a trial, the RT values were not recorded. The number of such a trial was $.4 \%$ of the total number of trials in Experiment 1 and $.5 \%$ in Experiment 2. These did not concentrate in particular conditions. (The standard deviations were $.8 \%$ in Experiment 1 and .9\% in Experment 2.)

2. Although the trend analysis failed to confirm, an inspection of Figures 2 and 3 seems to suggest a "dip and rise" in the masking functions. Using SOAs of 50,75 , and $100 \mathrm{msec}$ and combining the other variables, I performed a repeated measures analysis of variance to test the "dip and rise" in the masking function. The results were: $F(2,4)=10.01, p<.05$, in Experiment 1 , and $F(2,4)=4.37, p<.10$, in Experiment 2 . As these are a partial test, the significance level should be set at a level smaller than $5 \%$. Therefore, from the analyses, we can say only that the results of the analyses would suggest a trend of the "dip and rise."

(Manuscript received April 2, 1981; revision accepted for publication January 25, 1982.) 